\title{
EDITORIAL
}

\section{Syncope: what is the trigger?}

\section{R Hainsworth}

\begin{abstract}
Although a syncopal attack is frequently preceded by prodromal symptoms, sometimes the onset can be so abrupt that there is no warning at all. The switch in autonomic responses responsible for such an attack is quite rapid and dramatic, but the trigger for this remains one of the unresolved mysteries in cardiovascular physiology
\end{abstract}

$\mathrm{N}$ eurally mediated, or vasovagal, syncope is characterised by an abrupt fall in blood pressure resulting from widespread vasodilatation caused by a sudden cessation of sympathetic vasoconstrictor activity. There is no evidence in humans for an active neurally mediated vasodilatation, although there may be an element of reactive hyperaemia as an intense vasoconstriction suddenly stops. ${ }^{1}$ In addition to vasodilatation there is usually some cardiac slowing and this may be a small reduction in peak heart rate or, occasionally, prolonged asystole. The bradycardia is of less significance than the vasodilation $^{2}$ and preventing it by pacing does not usually prevent or even delay the onset of syncope. Vasovagal syncope usually occurs during orthostatic stress when not only is there pooling of something like 500-700 $\mathrm{ml}$ of blood in dependent vessels, but there is a progressive loss of a similar volume of plasma through dependent capillaries caused by the increased hydrostatic pressure. The overall effect of this stress is to reduce the rate of blood flow returning to the heart and consequently to reduce cardiac output. Blood pressure is normally well controlled mainly by arterial baroreceptors, which because of their exquisite sensitivity to changes in pulsatility, ${ }^{3}$ are able to induce vasoconstriction and tachycardia without a fall in mean pressure. Baroreflex sensitivity actually may be increased by interaction with other reflexes-for example, by reflexes excited by distension of the subdiaphragmatic veins. ${ }^{4}$ However, there seems to be a critical level of cardiac output, about half the supine level, when the normal control is interrupted and instead of vasoconstriction and tachycardia the efferent autonomic control switches to vasodilation and bradycardia.

A similar pattern of autonomic responses may also occur in susceptible individuals in response to emotional or painful stimuli-for example, during venepuncture. The emotional (or painful) stimuli and the effects of orthostasis seem to interact and there is a much higher incidence of syncope during head-up tilting in individuals with indwelling venous catheters. ${ }^{6}$

The syncopal attack is frequently preceded by prodromal symptoms, which may be present some minutes before there is a change in pressure. Sometimes, however, the onset can be so abrupt that there is no warning at all. The switch in autonomic responses is quite rapid and dramatic, but the trigger for this remains one of the unresolved mysteries in cardiovascular physiology.

\section{VENTRICULAR RECEPTORS ARE NOT THE TRIGGER}

Despite the now overwhelming evidence to the contrary, there is still a widely held view that the trigger for vasodilatation and bradycardia is provided by a paradoxical stimulation of cardiac ventricular receptors. The basis of this is the observation by Oberg and Thoren ${ }^{7}$ that some non-myelinated ventricular afferents could be excited when cardiac filling was low and sympathetic efferent nerves were strongly excited. This was said to elicit a Bezold-Jarisch reflex, a powerful depressor response. ${ }^{8}$ This mechanism was proposed despite the fact that any stimulus could only be short lived and baroreceptors would immediately be unloaded. There are several other problems with the ventricular receptor hypothesis. Firstly, only relatively few afferent nerves were excited in this way; most were less active. ${ }^{7}$ Secondly, powerful depressor responses to cardiac receptor stimulation have been shown to occur only to stimulation with foreign chemicals and not to mechanical stimuli. In bypassed near empty dog hearts, sympathetic stimulation did not cause the response. ${ }^{9}$ Thirdly, in humans, the dilator component of the reaction can be provoked following cardiac transplant, and therefore denervation. ${ }^{10}$ Finally, also in humans, the heart before syncope is not necessarily nearly empty nor powerfully contracting. It is apparent that some mechanism other than stimulation of ventricular receptors is needed to explain the sudden switch in autonomic activity.

\section{ALTERNATIVE HYPOTHESES}

It has long been realised that the brain must have a key role in the initiation of the syncope reaction. This could be by responding to a signal generated elsewhere, or by altering the processing by central pathways, or a combination of both. It is known that blockade of some central neurotransmitters can modify or prevent the onset of a vasovagal reaction. Work on animals has implicated opioid receptors, probably of the $\delta$ subtype, and administration of naloxone prevents the vasodilatation in several species. ${ }^{11}$ This effect of naloxone, however, has not been seen in humans, possibly because of the high doses needed. Another central neurotransmitter that appears to be involved is serotonin. Just before the onset of vasodilatation there is a surge in the serotonin concentration 
and use of centrally acting serotonin blockade may also prevent the dilatation. ${ }^{12}$

There are several hormonal changes coinciding with the onset of syncope. In particular, plasma vasopressin concentrations show a sudden and dramatic increase. ${ }^{11}$ Vasopressin, acting peripherally, is a vasoconstrictor agent, but acting centrally it greatly potentiates reflexes and in particular baroreflexes. ${ }^{13}$ If baroreflex sensitivity is greatly increased, relatively low levels of pressure could inhibit sympathetic efferent activity. It may explain an apparent increase in the response of baroreceptors whereby, when patients are returned to supine at the end of a tilt test, a single arterial pulse often seems to induce further vasodilatation and bradycardia, or even asystole.

Another possibility to consider in the initiation of syncope is whether a signal is obtained from a decrease in cerebral perfusion. There have been several claims that there is cerebral vasospasm at that time ${ }^{14}$ and speculation that this may initiate a chain of events leading to syncope. However, although a role for cerebral ischaemia cannot be excluded, the occurrence of cerebral vasospasm seems uncertain and may just be a consequence of pressure-flow non-linearity, with a critical closing pressure being approached. ${ }^{15}$

The findings by Mercader and colleagues, ${ }^{16}$ reported in a recent issue of Heart, are of particular interest as they provide an entirely different approach to the study of the events leading to syncope. They reported that in subjects who developed a vasovagal reaction, there was slow wave electroencephalographic activity from the left cerebral hemispheres. This was not seen in tilt negative subjects nor in a patient with postural hypotension who did not show the vasovagal reaction. It does, therefore, point to a change in central neuronal activity in the period leading to syncope. This work does not provide the answer as to what triggers the response, but it may provide a clue as to where we should be looking.

\section{PRESENT STATE OF KNOWLEDGE}

It is easier to state what does not trigger the switch in cardiovascular autonomic activity, than what does. It is certainly clear now that this switch cannot be attributed to stimulation of ventricular mechanoreceptors. Most recent evidence, including the article by Mercader and colleagues, ${ }_{1}^{16}$ indicates that the mechanism causing the switch lies within the brain itself. Pathways involving opioid and serotonin transmission have been implicated and very large changes in concentrations of cardiovascular acting hormones, and in particular vasopressin, have the potential to modify reflex responses. It is possible that the sensitivity of depressor reflexes becomes abnormally high, leading to a large and unusually prolonged vasodilatation and bradycardia. The switch is likely to involve a complex interaction of neuronal inputs, autonomic output, humoral effects, and ischaemia. However, its nature remains to be elucidated and the mechanism changing vasoconstriction and tachycardia abruptly to vasodilation and bradycardia still remain an unresolved mystery.

\section{REFERENCES}

1 Hainsworth R. Syncope and fainting. In: Mathias CJ, Bannister R. Autonomic failure, 4th ed. Oxford: Oxford University Press, 1999:428-36.

2 Hainsworth R. Heart rate and orthostatic stress. Clin Auton Res 2000;10:323-26.

3 Ead HW, Green JH, Neil E. A comparison of the effects of pulsatile and non-pulsatile blood flow through the carotid sinuses baroreceptors in the cat. J Physiol 1952;118:509-19.

4 El-Bedawi KM, Hainsworth R. Combined head-up tilt and lower body suction: a test of orthostatic tolerance. Clin Auton Res 1994:4:41-7.

5 Doe CPA, Drinkhill M, Myers DS, et al. Reflex vascular responses to abnormal venous distension in anaesthetized dogs. Am J Physiol 1996;271:H1049-56.

6 Stevens PM. Cardiovascular dynamics during orthostasis and influence of intravascular instrumentation. Am J Cardiol 1996;17:211-18.

7 Oberg B, Thoren P. Increased activity in left ventricular receptors during haemorrhage or occlusion of the caval veins in the cat. A possible cause of the vasovagal reaction. Acta Physiol Scand 1972;85:164-73.

8 Hainsworth R. Reflexes from the heart. Physiol Rev 1991;71:617-58

9 Wright C, Drinkhill M J, Hainsworth R. Reflex effects of independent stimulation of coronary and left ventricular mechanoreceptors in anaesthetized dogs. J Physiol 2000;528:349-58.

10 Fitzpatrick AP, Banner N, Cheng A, et al. Vasovagal reactions may occur after orthotopic heart transplantation. J Am Coll Cardiol 1993;21:1132-7.

11 Ludbrook J. Haemorrhage and shock. In: Hainsworth R, Mark A L, eds. Cardiovascular reflex control in health and disease. London: Saunders, 1993:463-90.

12 Grubb BP, Kosinski D. Serotonin and syncope: an emerging connection? Eur J Cardiac Pacing Electrophysiol 1996;5:306-14

13 Bishop VS, Hayworth JR. Hormonal control of cardiovascular reflexes. In: Zuker IH, Gilmore JP, eds. Reflex control of the circulation. Boca Raton: CRC Press, 1991:253-71.

14 Grubb BP, Gerard G, Roush K, et al. Cerebral vasoconstriction during head upright tilt-induced vasovagal syncope. A paradoxical and unexplained response. Circulation 1991;84:1157-64.

15 Carey BJ, Eames PJ, Panerai RB, et al. Carbon dioxide, critical closing pressure and cerebral haemodynamics prior to vasovagal syncope in humans. Clin Sci 2001;101:351-8.

16 Mercader MA, Varghese PJ, Potolicchio SJ, et al. New insights into the mechanism of neurally mediated syncope. Heart 2002;88:217-21.

\section{FROM BM JOURNALS}

\section{Prophylactic indomethacin can harm very low birthweight babies with severe CHD}

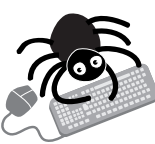

Please visit the Heart website [www. heartinl.com for link to this full article.

12 year review of very low birthweight babies with severe congenital heart disease (CHD) has dis covered that prophylactic indomethacin for patent ductus arteriosus can compromise a poor outcome still further. The risk would be significant if indomethacin treatment were to increase, claim the researchers.

Mortality was significantly higher in babies with CHD than without (40\%, 19/47 v 13\% 247/1973), and mortality due to CHD specifically was also significantly higher, at 32\% (15/47). Ventricular septal defect was the most common abnormality (27/47) but by itself did not cause death. Mortality rose still further once these cases were excluded. The second most common abnormality was coarctation of the aorta, present in eight babies. Five of these babies died, four who weighed $\leqslant 1000 \mathrm{~g}$ at birth. Six of the eight babies had indomethacin for patent ductus arteriosus before coarctation was diagnosed, and in three their condition got dramatically worse afterwards.

Sixteen babies were operated on, and seven died. Babies with coarctation had the poorest outcome: two died before their operation, and three of the six who had their abnormality corrected died; their birth weight was $\leqslant 1000 \mathrm{~g}$.

The study was based on data from the case notes of babies born between l January 1988 and 31 December 1999 in one hospital, $2020 \leqslant 1500 \mathrm{~g}$ and $760 \leqslant 1000 \mathrm{~g}$, who had severe CHD.

CHD occurs in 3-12/1000 live births. Its specific effect on very low birthweight babies has not been reported before.

A Archives of Disease in Childhood 2002;87:F31-F33. 